\title{
The speech therapy system for the formation of the phonemic processes of the junior pupils in the conditions of inclusion: the experience of teaching the students of the specialty of defectology
}

\author{
E.V. Matvienko ${ }^{1 *}, T . A$. Savelyeva $^{2}$, and A.S. Yarovaya ${ }^{3}$ \\ ${ }^{1}$ E.V. Matvienko Kuban State University, Krasnodar, Russia \\ ${ }^{2}$ T.A. Savel'eva Kuban State University, Krasnodar, Russia \\ ${ }^{3}$ A.S. Yarovaya Kuban State University, Krasnodar, Russia
}

\begin{abstract}
The article presents a detailed description of one of the areas of teaching the students of defectology receiving higher education - the study of the correctional technologies. The implementation of this direction plays an important role in the professional formation of the future specialists, as it contributes to the formation of students' general and special competences and the abilities to interact in the actual educational process. The presented system of the work aimed at the formation of the prerequisites for the formation of the processes of writing and reading of junior pupils, tested on the basis of the secondary schools in Krasnodar, implementing a system of the inclusive education. The effectiveness is confirmed by the quantitative and qualitative analysis of the data of the experiment with junior pupils.
\end{abstract}

\section{A problem statement}

The inclusive education has become a topical area of the pedagogical space due to the increase of the birth of the children with some disabilities. On this basis, the priority of the training of the specialists in the field of defectology is the formation of the students' knowledge and skills that help carry out professional activities in the conditions of the inclusive education and upbringing. Also, the current Federal State Educational Standard for the Primary Education defines a system of the new requirements for the results, the structure and the conditions of implementation of the basic educational program. The main attention of the standard is paid to the formation of the universal learning activities (ULA) in the primary period of the education, as it is the foundation for all further successful learning. One of the basic skills in forming the ULM is skill of writing and reading, the disorder of which is increasingly being elicited among the students of both special (correctional) schools and general education schools. This problem is one of the topical

\footnotetext{
*Corresponding author: prof-ped.gpa@mail.ru
} 
issues in the modern speech therapy. In this regard, the teaching of the students of the speech pathology should include the study of the correctional technologies aimed at the formation of the premeses for the formation of the process of writing and reading. The choice of the methods and the means of correction of these disorders within inclusive education defines the problem and the directions of the described research.

\subsection{The objective of the work}

The goal of the study is to develop a system of the logopedic work aimed for the correction of writing and reading processes of junior pupils who are in the conditions of inclusion.

In accordance with the purpose of the study the following tasks are formulated:

- to analyze the scientific literature on the formation and the correction of the process of writing of junior pupils in a secondary school;

- to determine the typology and the prevalence of the specific mistakes in the writing process;

- to evaluate the degree of the influence of the level of the development of the phonemic processes on the formation of the literacy skills;

- to determine the sequence of the directions of the logopedic work to correct the specific writing mistakes of junior pupils caused by the violation of the phonemic processes.

The hypothesis of the research is the assumption that the phonemic processes are one of the foundations for the formation of the literate writing skills. The lower the level of their formation of junior pupils is the more explicit their writing specific (dysgraphic) mistakes are, such as the substitutions of the letters when writing, the denoting the acoustically and articulatively similar sounds; the violations of the softness of the consonant letters in writing; the omission and the transposition of the letters and the syllables. The application in the correctional process of a system of the logopedic work on the formation of the phonemic processes, including the gradual formation of the phonemic hearing, the analysis and the synthesis, as well as the application of a differentiated approach in the logopedic sessions will reduce the number of the specific mistakes in the written work of the junior pupils.

The research methods: the theoretical analysis of the linguistic, psychological, pedagogical and methodological literature; the analysis of the medical and pedagogical documentation; the interview, the observation, the fact-finding the experiment; the quantitative and the qualitative analysis of the data of the fact-finding experiment and the statistical analysis of the results.

The research is based on the works of the domestic and the foreign authors who consider the problem of the writing disorders. According to S.S. Babkova, there is a difference in approach in the theory and the practice of speech disorders correction in Russia and abroad [1]. The foreign speech therapy uses clinical classifications of the speech disorders. According to the classification of the speech-language disorders C. Van Riper and L. Emerick, based on both syndromological and nosological approaches, all the speech and language disorders are divided into four categories: the articulation disorders; the voice disorders; the fluency disorders and the language disorders [2]. This classification does not correspond to the approaches of the domestic speech therapy. The speech correction abroad is aimed at the solving communicative problems, in the context of which all speechlanguage disorders are considered. Based on this, we can conclude that the foreign speech therapy considers the communication disorders as a criterion for the classifying disorders, rather than a symptom of a speech disorder, and the correction work is either medical or psychological in nature. For example, in the USA, in their practical work on the improvement of the fluency of the speech, the method of the reinforcement of the 
conditioned reflexes developed by Skinner and his followers, the work on the reduction of a threshold of the sensitivity to stuttering (desensitization), the explosive therapy (the stress reduction, through unpleasant situations), the use of the device Speech Easy ("Easy speech"), etc. are used [3]. In the domestic speech therapy, a comprehensive clinical and pedagogical and psychological-pedagogical classification of the speech disorders is used, which indicates a syndromological approach based on the etiopathogenesis of the disorders. Accordingly, the correction of these disorders is also complex. As we can see, many different approaches to the theory and the practice of the correction of the speech disorders are debatable and open, which is also complicated by the fact that, according to Y.O. Filatova, different countries adopt different designations of the speech disorders and different classifications, as well as there are national features of the professional vocabulary in the field of the speech therapy [4]. As for the impairment of the process of mastering written speech, today it is considered in various aspects: the psychological and pedagogical aspect (R.E. Levina, R.I. Lalaeva, L.N. Efimenkova, etc.) [5, 6, 7], the neuropsychological aspect (T.V. Akhutina, T.A. Fotekova, A.N. Kornev, etc.), [8, 9] and psycholinguistic aspect (A.R. Luria, V.K. Orfinskaya and others) [10,11]. Despite such close attention to this problem, the search for the effective ways of preventing and correcting this disorder is still going on. According to O.I. Azova, this is also due to the fact that specific disorders of the writing (dysgraphia) entail disorders in the mastering orthography [12], which often cause not only the persistent failure, but also the deviations in the formation of the child's personality. According to M.R. Lvov and D.B. Elkonin, one of the conditions for the formation of the writing skills is a developed phonemic hearing and a phonemic perception of the children who begin to acquire the literacy $[13,14]$. These phonemic processes are necessary for the development of the orthographic skills, i.e. the skill of mistake-free writing.

The practical research was carried out on the basis of the secondary schools in Krasnodar which implement a system of the inclusive education. The experiment was attended by the second-grade pupils in the number of 148 people, aged eight to ten years.

\section{Results of the research}

The practical study consisted of several stages.

The first stage was the development of a modified technique for the examination of the phonemic processes of the junior pupils. To implement this stage we studied and analyzed the methods of the examination of the following authors: L.F. Spirova, E.N. Russian, O.I. Azova, I.N. Sadovnikova, T.V. Akhutina, T.A. Fotekova, R.I. Lalaleva, L.V. Venidiktova and highlighted the areas of the phonemic processes examination and determined the content of each of them.

The second stage was the selection and the primary examination of some children in the experimental group. During this stage we got acquainted with the writing works of the second grade children and selected the students with unformed writing process skills. Also, the dictation test was made in the second grade in order to clarify the symptomatology and severity of the impairment of the literacy skill.

The third stage was the logopedic examination, the description of the features of the formation of the phonemic processes of the children participating in the experiment. At this stage, the second-grade pupils with writing disorders were examined in order to identify the causes of the unformed skills, as well as all the other pupils in order to determine the influence of the level of the phonemic processes development on the level of the formation of the writing process.

The fourth stage was the designing a system of the logopedic work on the formation of the phonemic processes of junior pupils. During this stage classical methods of correctional 
work in the studied direction were studied by T.B. Filicheva, G.V. Chirkina, I.A. Cheveleva, I.N. Sadovnikova, R.I. Lalaeva as well as developments by modern authors: E.V. Mazanova, L.N. Efimenkova, I.A. Lukina, I.I. Nikkenen, A.M. Goncharova and O.V. Eletskaya. The studied material made it possible to draw up a long-term work plan for the formation of the phonemic processes of the children of primary school age, including areas of the work with recommended tasks for each of them, as well as to select the didactic material for the implementation of the correctional and developmental work in the selected areas of work.

The implementation of the first stage began with the development of a modified technique for studying phonemic processes of junior pupils. Having studied the available literature on this issue, we identified six areas of the research of the considered component of the speech:

- the study of the ability to recognize and to differentiate the non-speech sounds: the recognition of the non-speech sounds and the sound localization;

- the study of the ability to perceive and to reproduce rhythms: the perception and the reproduction of the rhythms, the study of the auditory-motor coordination;

- the study of the ability to recognize and to distinguish the syllables and the words similar in the sound composition: the reproduction of the syllable series, the identification of the correct and distorted pronunciation of the words, the identification of the words similar in sound; perception and reproduction of the words that differ acoustically and articulatively by the similar sounds;

- the study of the elementary skills of the phonemic analysis: the identifying a sound in the background of a word, the determining the place of a sound in a word and the selection of the words with a specific sound;

- the study of the complex forms of the phonemic analysis: the determining of the sequence of the sounds in a word, the determining the number of the sounds in a word and the making a sound model of a word;

- the study of phonemic synthesis: the making of the words out of the sounds in an undisturbed sequence and the making of the words out of the sounds in a disturbed sequence.

The protocol of the research on the allocated directions with the detailed instructions for each task was made up. The illustrative materials for the protocol was selected from the following logopedic examination albums [15-20].

The reseach of the state of the writing skills began with the analysis of the workbooks of second-grade pupils and an auditory dictation. Analyzing the written works, we recorded the presence of the spelling and specific (dysgraphic) mistakes in the protocol. This analysis allowed us to divide the experimental group into three subgroups based on the level of the literacy skill formation as described by A.V. Yastrebova:

1) the group of children who have formed the skill of the literate writing. In the works of these children there are no specific (dysgraphic) mistakes, there is a small number of spelling mistakes corrected mainly by the pupils themselves;

2) the group of children with insufficiently formed literacy skills. In the works of these children there is a small number of specific (dysgraphic) mistakes, which are found and corrected mainly by pupils themselves and spelling mistakes connected to the studied rules are found;

3) the group of children with unformed literacy skills. In the works of these children there is a large number of both spelling and specific (dysgraphic) mistakes, which are not found and not corrected by pupils themselves.

As a result of this analysis 42 out of 148 second grade pupils have formed the literacy skills (28\%), 68 pupils have insufficiently formed skills (46\%) and 38 pupils have unformed skills $(26 \%)$. The obtained data show the need for the corrective and 
developmental work with $50 \%$ of the pupils who have insufficiently formed and unformed literacy skills. Based on the recommendations of A.V. Yastrebova with pupils who have insufficiently formed writing skills, it is recommended to conduct the corrective work within the educational process by including additional corrective exercises in the structure of the Russian language and the literature reading lessons. With pupils with the unformed writing skills, additional corrective lessons are organized, and these children are the subject of the enrollment in the logopedic unit of the general education institution. Further experimental work was conducted with some children who showed the unformed literacy skills at the first stage. The goal of the further study was to determine the typology of the specific writing mistakes and to identify the mechanism of their formation.

The analysis of the typology of the specific mistakes showed that the following types of the mistakes predominate in this group of junior pupils: the mistakes of the sound analysis, in particular the omissions of the vowel and consonant sounds, the transposition and the addition of the letters and the syllables in the word structure; mistakes related to the mixing of the letters denoting sounds close in the acoustic and articulatory features (the most common mixed pairs - $[\mathrm{P}]-[\mathrm{B}],[\mathrm{K}]-[\mathrm{G}],[\mathrm{S}]-[\mathrm{Sh}],[\mathrm{Sh}]-[\mathrm{Tsh}$ '], [S]-[Ts]); the spelling mistakes (the most numerous - the spelling of a paired consonant and the vowel free in the root of the word).

The preliminary data of the research indicate the predominance of the mistakes related to the violation of the phonemic processes in the study group. In order to make a detailed comparative analysis of the mistakes in writing, we conducted an auditory dictation. The most frequent mistakes were the absences of the vowel and consonant letters (for example, ("ws" - was, "opn" - open, "frsh" - fresh, "blming" - blooming"("beauiful" - beautiful, "er" - her),the omissions and the transpositions of the syllables; the addition of letters (for example, "warme" - warm, "scchoolchildren" - schoolchildren), the indicating persistent violations of syllabic and phonemic analysis and synthesis skills. The missing of the vowel letters were observed both in the middle and in the end of the word, which, in our opinion, is due to the fact that in the process of the writing a word pupils can better identify a consonant sound than the vowel sound that follows it. The weakness of this mechanism may also be due to the low level of the stability and the switchability of the attention. Just as frequent there were the mistakes in the delimitation of the speech units: the absence of a point at the end of a sentence, the replacement of a capital letter by a lowercase letter at the beginning of a sentence and the fused spelling of the words. More often the pupils combined two sentences into one, which was reflected in the grammatical design of the sentences, there were mistakes of the word agreement, at the level of the word combinations, pupils also allowed the omission of the words. All this testifies to the fact that pupils have impaired the clear perception of the speech stream, they cannot distinguish the complete units of the speech in the semantic sense. The mistakes in the fusion writing of the prepositions with, other words made by the pupils indicate that they do not distinguish the preposition as a separate word, do not know the semantics of the prepositions. These mistakes may also indicate the difficulties in the semantic decoding in the process of the reproduction of the speech stream and the lack of the formation of the arbitrary regulation of actions. The mistakes were also noted for the mixing of the letters by the acousticarticulatory and optical similarity. In the written works, there was also a variety of the mistakes in the mixing of letters by acoustic-articular similarity, which indicates a persistent lack of phonemic hearing, sound-letter associations, and a low level of volume and distribution of attention. The most common were: the violation of the differentiation of the vowels, the mixing of the whistling sounds within the phonetic group, the violation of the differentiation of the paired consonant sounds. 
Among the children with the unformed writing skills, $65 \%$ have pronounced specific errors, according to our assumption, due to the lack of the phonemic processes. The ascertaining stage of the research was continued with this group of the children.

The next stage of the work was a logopedic examination of the children in order to determine the causes of the writing disorders. The examination was conducted with each child individually, for each child a protocol of the phonemic processes research was filled. The analysis of the examination protocols allowed us to give a qualitative-quantitative assessment of the results of the study of the phonemic processes of the children with a low level of the formation of the literate writing skills. There is a detailed analysis of the results of the examination per subject in the table 1 .

Table 1. The example of the analysis of the results of a speech therapy examination of the children with a low level of literacy skills.

\begin{tabular}{|c|c|c|}
\hline Name & $\begin{array}{l}\text { Writing mistakes } \\
\text { (dictation analysis) }\end{array}$ & $\begin{array}{l}\text { The features of the development of the phonemic } \\
\text { processes }\end{array}$ \\
\hline \multirow[t]{6}{*}{ Miron N. } & \multirow{6}{*}{$\begin{array}{l}\text { The mistakes in the } \\
\text { sound analysis: the } \\
\text { omission of vowel } \\
\text { letters ("ws" - was, } \\
\text { "opn" - open, "frsh" - } \\
\text { fresh, "blming" - } \\
\text { blooming), the } \\
\text { omission of consonant } \\
\text { letters ("beauiful" - } \\
\text { beautiful, "er" - her). } \\
\text { The delimitation of } \\
\text { the speech units: fused } \\
\text { prepositions with } \\
\text { words ("behindit" - } \\
\text { behind it, "onit" - on } \\
\text { it), a small letter at the } \\
\text { beginning of a } \\
\text { sentence. The mixing } \\
\text { letters by the acoustic } \\
\text { and articulatory } \\
\text { similarity: violation of } \\
\text { the differentiation of } \\
\text { vowel sounds [e] - [i] } \\
\text { ("rever" - river), [a] - } \\
\text { [e] ("pletforms" - } \\
\text { platforms), consonant } \\
\text { sounds [c] - [ts], [p] - } \\
\text { [1]. } \\
\text { The lexical and } \\
\text { grammatical mistakes: } \\
\text { the omission of the } \\
\text { words in the structure } \\
\text { of the sentence, the } \\
\text { mistakes of the word } \\
\text { formation ("frash" - } \\
\text { fresh, "worm" - } \\
\text { warm). }\end{array}$} & $\begin{array}{l}\text { 1. The examination of the ability to recognize and to } \\
\text { differentiate non-speech sounds. Distinguishes the distant } \\
\text { sounding non-speech sounds, differentiates the close } \\
\text { sounds at the second attempt. The determining of the } \\
\text { localization of a sound is difficult; after repeated } \\
\text { attempts, he only detects upward or in front. }\end{array}$ \\
\hline & & $\begin{array}{l}\text { 2. The study of the ability to perceive and to reproduce } \\
\text { the rhythms. The reproduction of the simple rhythms is } \\
\text { accessible. The reproduction of the difficult rhythms is } \\
\text { partially accessible after repeated listening. }\end{array}$ \\
\hline & & $\begin{array}{l}\text { 3. The examination of the ability to recognize and to } \\
\text { distinguish the syllables and the words similar in the } \\
\text { sound composition. The violation of the distinction of the } \\
\text { syllables and the words containing similar acoustic and } \\
\text { articulatory sounds: [s]-[s], [t]-[d], [sh]-[tsh'], [s]-[ts]. } \\
\text { When analyzing the sentences, there are numerous errors } \\
\text { associated with the violation of the differentiation of the } \\
\text { phonemes. The recognition of the correct sound of a } \\
\text { word is accessible with the help of a picture, when } \\
\text { perceiving by ear mistakes are made, which are corrected } \\
\text { independently. }\end{array}$ \\
\hline & & $\begin{array}{l}\text { 4. The examination of the elementary skills of the } \\
\text { phonemic analysis. The allocation of the sounds and the } \\
\text { identification of the place of the sound in general is } \\
\text { available, the erroneous execution of the task in words } \\
\text { where there are sounds which distinction is difficult. The } \\
\text { matching words to a certain sound is available only with } \\
\text { the reference pictures, do not pick up independently. }\end{array}$ \\
\hline & & $\begin{array}{l}\text { 5. The examination of the complex forms of the phonemic } \\
\text { analysis. Determines the sequence and number of the } \\
\text { sounds only in the words with three letters. Makes a } \\
\text { sound model with numerous mistakes, after pointing } \\
\text { them out can partially correct them. }\end{array}$ \\
\hline & & $\begin{array}{l}\text { 6. The examination of the phonemic synthesis. } \\
\text { Composing the words from the letters is accessible only } \\
\text { from the sounds in an undisturbed sequence. Making the } \\
\text { words out of the sounds in a broken sequence is not } \\
\text { accessible even with the help of the pictures. }\end{array}$ \\
\hline
\end{tabular}


The presented data reflects the typology of the writing process disorders and the causes of these disorders. The reseach showed a rather low level of the formation of all identified components of the phonemic system in the experimental group: the ability to recognize and to differentiate non-verbal sounds, the ability to perceive and to reproduce rhythms, the ability to recognize and to distinguish the syllables and the words that are similar in sound composition, the presence of the elementary skills of the phonemic analysis, the presence of skills of the complex forms of the phonemic analysis and the phonemic synthesis.

In order to confirm or refute our assumption that the phonemic processes are one of the foundations for the formation of the literate writing skills and the lower the level of their formation of junior pupils is the more explicit in their writings specific (dysgraphic) mistakes are such as the substitutions of the letters when writing, denoting of acoustically and articulatively similar sounds; the violations of the marking the softness of the consonant letters in writing; the omission and the transposition of the letters and the syllables, we decided to study the level of the formation of the phonemic processes of all second graders who took part in the beginning of the experimental work. During the examination the protocol was also filled for every child, the level of the formation of each component included in the phonemic processes was deduced. In the work we will present generalized data for the whole group under study. As a result, the average level of the development of all the examined components was deduced and correlated with the levels of the formation of the writing process of the children in the beginning of the experimental work, thus that the higher the level of the phonemic processes development is the higher the level of the literate writing skill formation. We have proved that phonemic processes are one of the conditions for the formation of the written speech and that their violation leads to dysgraphia.

The final stage of this research involved the development of a system of the logopedic work aimed at the correcting writing errors caused by the violation of the phonemic processes. When developing the components of the logopedic technology we relied on V.A. Slastenin's definition of the "pedagogical technology", so our development includes the definition of the principles of the correctional work, the long-term planning with a detailed description of the stages of work, the selection of a system of the tasks, some exercises and the didactic material for implementing the long-term planning. The traditional and the innovative methodological approaches formed the basis of the technology of the forming phonemic processes. The theoretical and the methodological basis was the principles developed in the defectology and the speech therapy by L.V. Vygotsky, R.E. Levina and others:

- the principle of the development and the reliance on the speech ontogeny. The perspective plan provides step-by-step work on the formation of the phonemic processes, beginning with the development of the skills such as distinguishing non-verbal sounds, intonations, timbre, voice strengths and then proposing the work on the speech sounds in the sequence in which they appear in the ontogenesis, then working on the skill of the phonemic analysis and the synthesis;

- the principle of a differentiated approach. The developed system allows us to design the individual and the subgroup work, taking into account the disorders of each child;

- the principle of the comprehensiveness of the correctional influence. Through making the tasks for the formation of the phonemic hearing, the development and the improvement of all components of the speech system is underway, especially the skill of the language analysis and the synthesis.

The speech therapy system for the formation of the phonemic processes of the junior pupils includes 5 stages of the work:

1) preparatory:

- the recognition of the non-speech sounds; 
- the perception and the playback of the rhythms;

- the perception and the reproduction of the intonation and voice modulation by volume and timbre.

2) the formation of the phonemic hearing:

- the distinguishing words with the similar sound composition;

- the highlighting vowel sounds in the background of the word;

- the differentiation of the syllables;

- the introduction to the consonant sounds.

3 ) the differentiation of the sounds close in the different acoustic and articulatory features:

- the differentiation of the sounds similar in the articulation features;

- the differentiation of the sounds similar in the acoustic features.

4) the formation of the simple forms of the phonemic analysis:

- the highlighting a sound in the background of a word;

- the identifying the first and the last sounds in a word;

- the determining the place of a sound in a word.

5) the formation of the complex forms of the phonemic analysis and synthesis:

- the formation of the phonemic analysis with the reliance on aids and external actions;

- the formation of the actions of phonemic analysis mentally;

- the formation of the ability to form words from the sounds presented in an unbroken and broken sequence.

The system of the logopedic work presented in the table 3 will allow to form the skill of the literate writing of the junior pupils, the reasons of the violation of which are the unformed phonemic processes. The development of the selected areas in the first grade will prevent the occurrence of the errors connected to the lack of the phonetic principle of writing, such as replacement of letters when writing of denoting acoustically and articulatively similar sounds, the violation of consonant softness marking in the writing; the omission and the transposition of the letters and syllables.

\section{Conclusions}

Thus, the research has revealed a) a relatively large percentage of children with unformed literate writing skill distinguish among the pupils of the mass school $(24 \%)$; b) the main reason for this disorder is the low level of the development of the phonemic processes; c) phonemic process's violations are expressed in the omissions, the letter substitutions, the replacement of the letters of acoustically and articulatively similar sounds, the violation of consonant softness marking; d) the higher the level of the phonemic processes development is the higher the level of the literacy skill formation; e) the formation of the phonemic processes should be performed step-by-step: starting with the ability to differentiate nonspeech sounds, then the ability to get the sound in the background of a word is refined and this process ends with the development of the ability to make a complete sound-letter analysis of a word f) for more effective corrective work it is necessary to include some exercises that contribute to the formation of the psychological base of the speech, in particular some exercises for the development of the auditory attention and memory.

This suggested system of the speech therapy work can be recommended to the specialists working with the junior pupils who are in the system of the inclusive education, as well as to the students of the speech pathology studying in the field of "Special (defectological) education". 


\section{References}

1. S.S. Bobkova, The difference in the approaches in the theory and in the practice of speech impairment correction in Russia and abroad, Special Education, materials of the XIII international scientific and practical conference, 14-18 (2017)

2. C. Van Riper, Speech Correction: An Introduction to Speech Pathology and Audiology, 8 (1990).

3. S. Xia, T. Jiang, J. Kalinowski, M. Rastatter, The first self-contained in-the-ear device to deliver altered auditory feedback, ApplicatApplications for stuttering. Annals of Biomedical Engineering, 31, 233-237 (2003)

4. Y.O. Filatova The Rhythm of the speech and movements of the children: theoretical and applied problems of speech therapy, 233-237 (2012)

5. R.E. Levina, Speech and writing disorders of the children. Selected works, 221 (2005)

6. R.I. Lalaeva. The diagnostics and the correction of reading and writing disorders of the junior pupils, 176 (2003)

7. L.N. Efimenkova, The correction of the errors caused by lack of phonemic perception, 132 (2017)

8. T.A. Fotekova, T.V. Ahutina, The neuropsychological diagnosis of the speech pathology of the children, School psychologist, 37, 3 (2001)

9. A.N. Kornev, Reading and writing disorder of the children (diagnostics, correction, prevention, 286 (2004)

10. A.R. Luria, Psychological Heritage, 431 (2003)

11. V.K. Orfinskaya, On the education of the phonological representations in the junior school age. Psychology of speech, 125 (1997)

12. O.I. Azova, The logopedic survey of the junior pupils, 64 (2011)

13. M.R. Lvov, The methodology of the speech development of the junior pupils, 238 (2003)

14. D. Elkonin, The stages of the forming of the action of reading: phonemic analysis of the words, 64 (1976)

15. O.I. Azova, The logopedic examination of the junior pupils. Diagnostic kit, 48 (2008)

16. G.A. Volkova, An album for the study of the phonetic and phonemic side of the speech for preschool children, 144 (2006)

17. I.A. Smirnova, The logopedic album for the examination of the phonetic-phonemic system, 54 (2018)

18. I.A. Smirnova, The logopedic album for the examining of the ability to read and to write, 64 (2017)

19. T.N. Volkovskaya, An illustrated technique of a speech therapy examination, 144 (2004)

20. O.B. Inshakova, An album of a speech therapist, 279 (2015) 